\title{
Multi-Source Spatial Data Distribution Model and System Implementation
}

\author{
Jing Liu' ${ }^{1}$ Xiancheng Mao² \\ ${ }^{1}$ School of Geosciences and Info-Physics, Central South University, Changsha, China \\ ${ }^{2}$ MOE Key Laboratory of Metallogenic Prediction of Non Ferrous Metals, Central South University, Changsha, China \\ Email: liujing8280@163.com,xcmao@126.com
}

Received November 12, 2012; revised December 15, 2012; accepted January 16, 2013

\begin{abstract}
The Multi-source spatial data distribution is based on WebGIS, and it is an important part of multi-source geographic information management system. a new multi-source spatial data distribution model is proposed on the basis of multisource data storage model and by combining existing map distribution technology, The author developed a multi-source spatial data distribution system which based on MapGIS K9 by using this model and taking full advantage of interfacecode separating thinking and high efficiency characteristic of .net, so high-speed distribution of multi-source spatial data realized.
\end{abstract}

Keywords: Multi-Source Spatial Data; Distribution Model; WebGIS; MapGIS K9

\section{Introduction}

The spatial data distribution is an important part of WebGIS. At present, the content of map distribution study includes: the ways of map distribution [1,2], the technology of map distribution [3-5], how to use current soft middleware to develop a map distribution system. These studies mainly focused on a single map distribution and they do not describe the multi-source spatial data distribution in detail. So a multi-source spatial data distribution model which based on multi-source data storage model is proposed in the paper, and as an example of the model, the author developed a multi-source spatial data distribution system by using MapGIS K9 middleware, which achieve high-speed distribution of multi-source spatial data.

\section{Multi-Source Data Model}

The multi-source data model includes multi-source data storage model and multi-source spatial data distribution model. The latter is built on the foundation of the former, so their relationship is inherited.

\subsection{Multi-Source Data Storage Model}

Most data for applications has the nature of multiple sources, such as geology application. They can be seen as the performance of classification diversity. The multisource data integration management is the integrated management of various classification, which is reflected in the data storage. By using some documents about various aspects of multi-source heterogeneous data integration for reference [6-11], and combining with existing models, a general multi-source data storage model is proposed.

\subsubsection{Classification Element}

The data with nature of multiple-sources can be defined as an abstract classification, and the data itself regarded as an attribute in it. The abstract classification has three attributes, that is identification, metadata and entity. On the whole, the abstract classification is called classification element, which is a triple group. It is defined as:

Data Element $=<$ Type, Metadata, EntityData $>$

Type is an identifier of data classification, which used to represent a type of data element. In geographic database application, type may stand for a certain classification of scale or classification of sheet line system. Metadata is the data which can display the feature of classification element. Entity Data is the content of the dataelement, which descript a certain classification in detail. It may represent spatial data, attribute data, document and so on.

\subsubsection{Classification Tree}

Classification Tree is a tree structure based on classification element, which describes the characteristics of data and data between inheritance and containment relationships. Obviously, the classification tree is the abstract form of data classification system, and we can get the multi-source classification after instantiating the tree. 
Figure 1 shows an example of geographic data classification.

\subsection{Multi-Source Spatial Data Distribution Model}

It is convenient for existing technology to distributing a single map, which only requires administrator transmitting some parameters (data path, symbol path etc.) to the middleware of map distribution. As to the mass of multisource spatial data, if we adopt the multi-source data storage model above and combine with the single map distribution technology, we can easily complete the multisource map distribution. The procedure is as follows: step 1: organizing the multi-source data as classification tree. Step 2: selecting spatial data from the classification tree. Step 3: get parameters from the selected spatial data and transmit them to the software of distribution map. step4: complete map distribution by using the software. Step 5: obtain the distributed parameters and save them to database. The purpose of step 5 is to browse the final distributed map, for the middleware, which help users get the web map, need the distributed parameters. After completing all the steps, the spatial data of a classification tree node is mapped to a distributed map and all of them form a map tree. The multi-source spatial data distribution model is shown as Figure 2.

\subsubsection{Composition of the Model}

The model is composed of input storage, processing and output storage. The input is the beginning and the output is the last, both of them store in a way just like the classification tree above. The processing is the core of the whole procedure, which responsible for accepting multisource data and selecting spatial data from the input storage, completing map distribution by using middle-

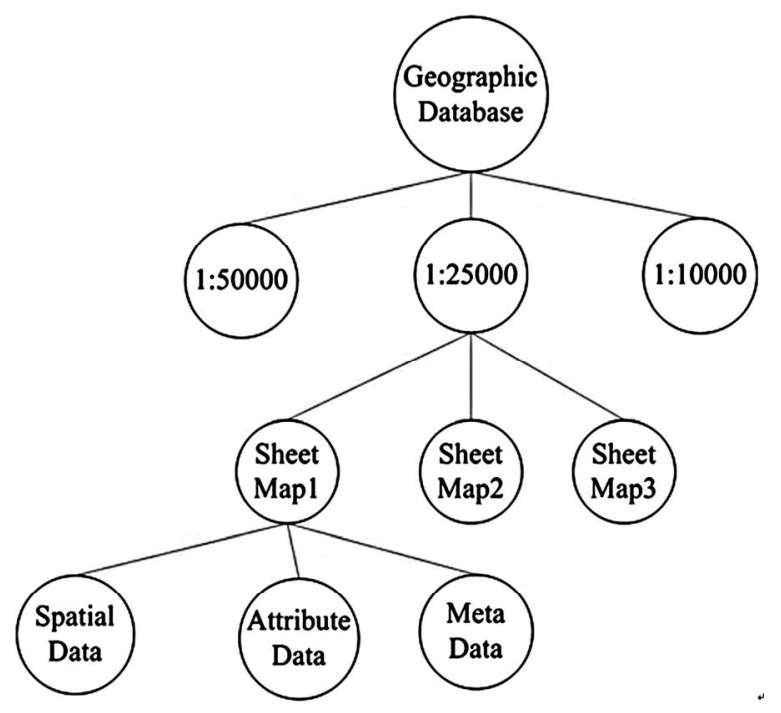

Figure 1. Geographic database classification tree. ware and giving the final map tree to the output storage. It includes artificial interaction process and automatic map distribution process.

\subsubsection{Functions of the Parts}

Input and output storage: all the data for applications are stored as classification tree. For example, the geographic data will be organized as the way that Figure 1 shown. The two storages will be realized in the database design part, by using children-relationship method in reference 10.In fact, the Input storage is mapped to the output storage, for the output storage is making up by the map tree, which is organized as classification tree yet.

Processing: it includes artificial interaction and automatic map distribution. In the former process, the administrator will check the validity of the spatial data. If the data meets the requirements, the administrator will post it to the latter course. Otherwise, the administrator will inform the user who uploads the data of changing the data and uploading again. In the automatic map distribution process, a third part software will be used to realize the distribution of the map. What the essence of this process is to establish the mapping between the original spatial data and the map.

\section{Map Distribution System}

As an application instance of multi-source spatial data distribution model, we take C\# and MapGIS K9 to develop a multi-source spatial data distribution system, which is an important part of multi-source geographic information system which is based on WebGIS. The distribution system can help administrators to complete a variety of spatial data distribution easily. In the development, we took MapGIS K9 class library and MapGIS K9-IMS middleware for further development, the former mainly helps administrators import the spatial data to the database of MapGIS, and the latter provide administrators with the interface of automatic map distribution.

\subsection{Structure}

Figure 3 shows the structure of the distribution system. We take geological application for example, so the type of the database is mainly about geological database.

\subsection{Database Design}

The multi-source data storage model has been realized through database design, that is, the construction of the classification tree. Taking geological application for example, this application includes variety of database, such as geographic database, geological work progress database, drilling database etc. during the database design progress, all the database should have their own database table structure. If we extract the shared fields from the 


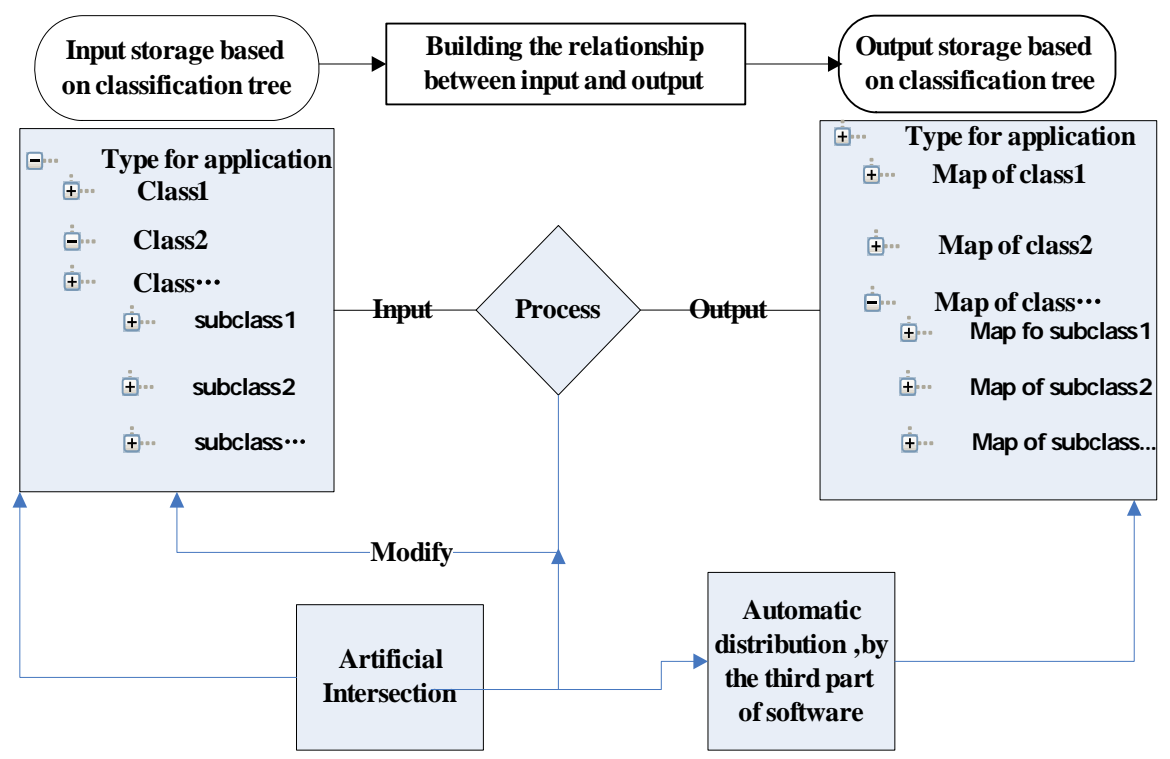

Figure 2. Multi-source spatial data distribution model.

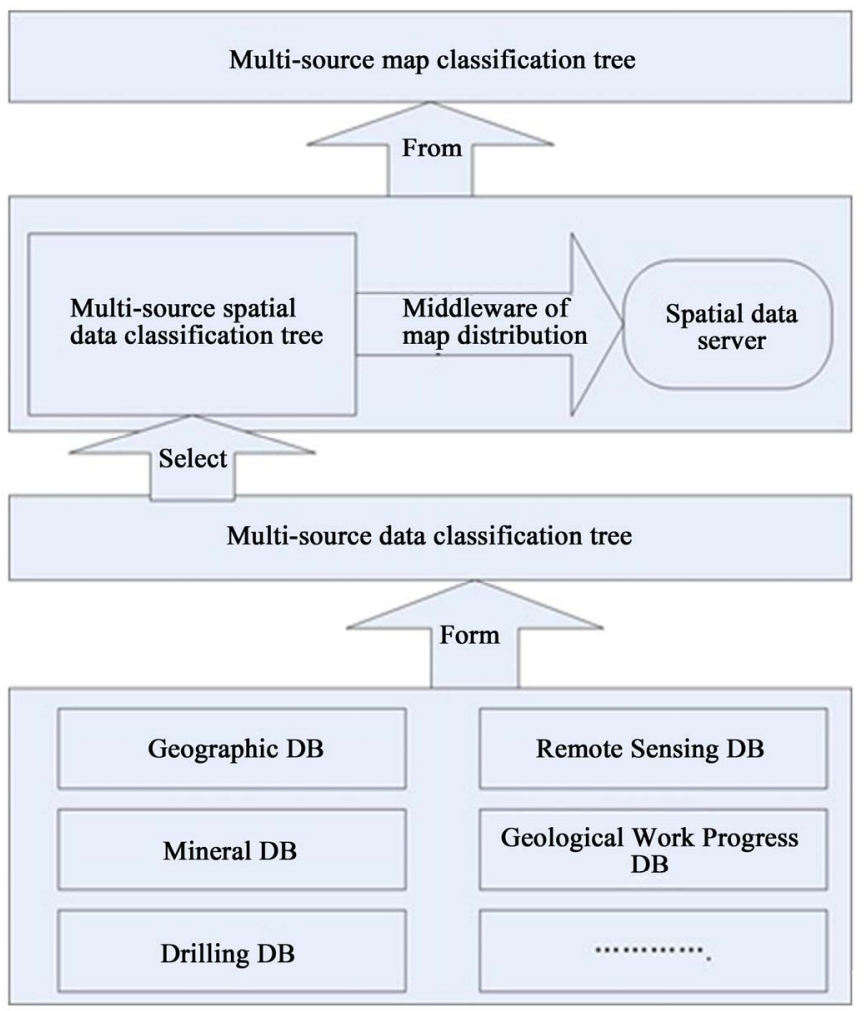

Figure 3. Structure of multi-source spatial data distribution system.

tables, a general template of all classification trees will be formed. For that, it is convenient to design other types of database, which only need to add some certain fields to the templates. The followings are templates extracted from the tables. Table 1 shows the structure of the classification tree, which store the classification elements. The ID and FatherID field can be found in the table, and we can create classification trees by recursion through these two fields. Table 2 stores the map parameters of using MapGIS K9 distributing the multi-source spatial data.

When creating the database for applications, you only need to add some fields that related to the specific application to the templates. Such as geographic database in- 
Table 1. Table of classification tree.

\begin{tabular}{|c|c|c|c|}
\hline Name & Type & Constraint & Note \\
\hline ID & NUMBER & Primary & $\begin{array}{l}\text { Identity of the tree } \\
\text { node }\end{array}$ \\
\hline Name & VARCHAR2 (50) & Not null & No repeated \\
\hline FahterID & NUMBER & Not null & $\begin{array}{l}\text { Father identity of } \\
\text { current node }\end{array}$ \\
\hline DataType & VARCHAR2 (4) & Not null & $\begin{array}{c}\text { Type of } \\
\text { classification }\end{array}$ \\
\hline
\end{tabular}

Table 2. Table of map distribution.

\begin{tabular}{|c|c|c|c|}
\hline Name & Type & Constraint & Note \\
\hline ID & NUMBER & Not null & $\begin{array}{l}\text { Refer to the ID of table of } \\
\text { classification tree }\end{array}$ \\
\hline DataID & NUMBER & Index & $\begin{array}{l}\text { Constitute primary key } \\
\text { with ID }\end{array}$ \\
\hline MapName & $\begin{array}{l}\text { VARCHAR2 } \\
(50)\end{array}$ & Not null & The name of the map \\
\hline MAPPath & $\begin{array}{l}\text { VARCHAR2 } \\
(500)\end{array}$ & & $\begin{array}{l}\text { The storage path of the } \\
\text { map }\end{array}$ \\
\hline
\end{tabular}

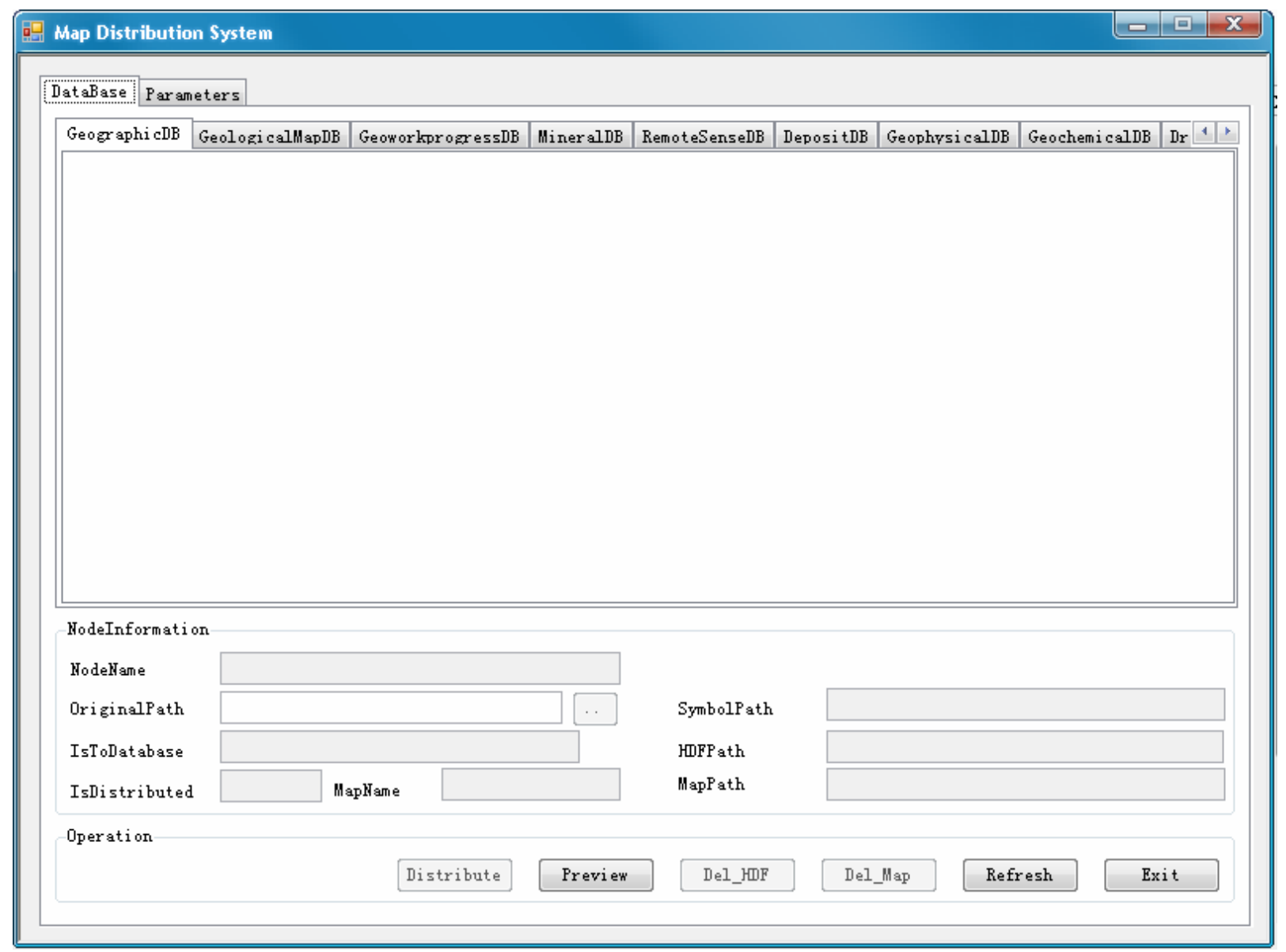

Figure 4. The interface of multi-source spatial data distribution system.

cluding scale information and project information, therefore you just need to add scale field and project type field to the table of map distribution.

\subsection{Detailed Design}

It is convenient to distribute spatial data by MapGIS K9, which only requires users to import the original spatial data into the database of MapGIS K9 and create the file with the suffix of map in the distributed folder of MapGIS IMS. As a matter of fact, the process of map distribution includes two steps, the one is putting the spatial data to the database of MapGIS, the other is creating map in the distributed folder of MapGIS IMS. The system is designed by using object-oriented approach, so a wide range of classes will be defined. Such as class of layers, class of map, class of map operation and so on. The class of layers and class of map are the key classes, which complete these two main steps of map distribution.

Class of layers: in order to import spatial data into MapGIS successfully, the method provided by mc_basXcls7Lib.dll from MapGIS K9 will be encapsulated in the class. MapGIS K9 can support the data of 6x and $7 \mathrm{x}$ format, so we should take the difference between 


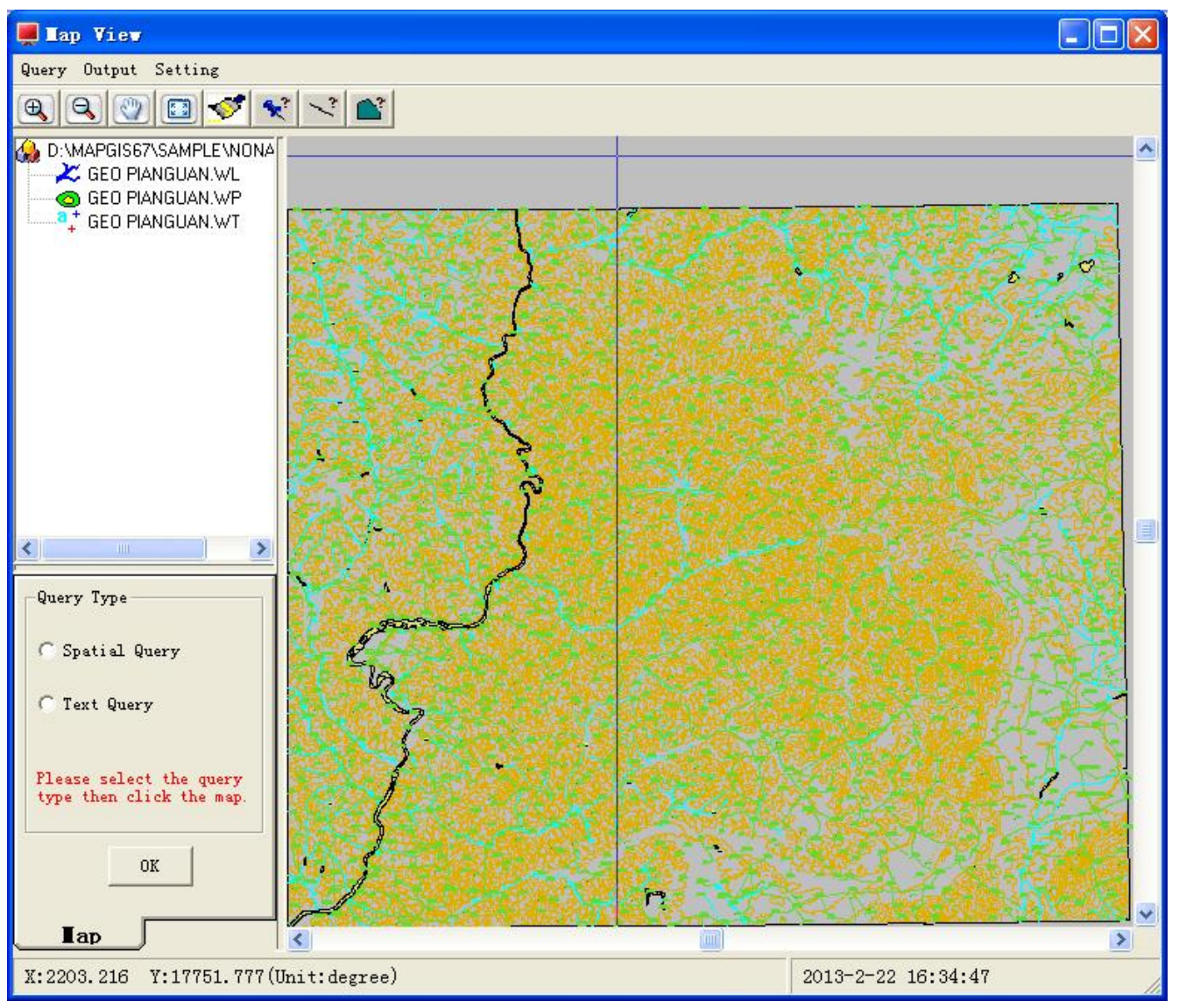

Figure 5. The renderings of map distribution.

the two into consideration. The 7x format is the standard one that MapGIS K9 can support, which can help us easily complete the importing, just calling the method from the mc_basXcls7Lib.dll. To the other condition, it need to change the $6 \mathrm{x}$ data format to $7 \mathrm{x}$ data format, because $6 x$ is the old version data format of MapGIS6.7.

Class of map: The method of creating map and operating map parameters are included in this key class. the former achieved the functions of creating map in the distributed folder of MapGIS K9 and organizing the hierarchy of the maps as the original spatial data. The latter achieved the function of operating the map parameters in the table of map distribution, such as add parameters to the table, modify parameters, delete parameters from the database.

Figure 4 shows the main interface of multi-source spatial data distribution system, and Figure $\mathbf{5}$ shows the final renderings of the map distribution.

\section{Conclusion}

The data for applications has the characteristics of great variety, huge quantity, and complex data format. How to organize and distribute these data fast through web have directly impact on the progress of resource and informa- tion sharing. A new multi-source spatial data distribution model is proposed in the paper by using multi-source data storage model and combining existing map distribution technology. At last, we developed a multi-source spatial data distribution system on the basis of the model and MapGIS K9, which is strong, flexible, and achieved the rapid distribution of the map.

\section{REFERENCES}

[1] L. L. Geng, Z. Li and N. Liu, "Research into Dynamic Map Service Management Object Model [J]," Journal of Zhejiang University (Science Edition), Vol. 37, No. 4, 2010, pp. 471-481.

[2] Z. K. Xu, D. Q. Liu and Z. J. Lin, "Study on Distribution Technology of Dynamic Thematic Map Based on WebGIS [J]," Science of Surveying and Mapping, Vol. 31, No. 3, 2006, pp. 104-105.

[3] H. W. Yan, T. Wu and L. F. Fei, "The Research of Network Map Publication Technology Based on Flash and Its Application [J]," Science of Surveying and Mapping, Vol. 30, No. 3, 2005, pp. 73-74.

[4] Q. Z. Geng, "Design and Development of Spatial Data Release System of Dam in China Based on WebGIS [J]," Water Resources and Power, Vol. 25, No. 6, 2007, pp. 44-47. 
[5] G. I. Thrall, “Digital Map Publishing [J],” Vol. 15, No. 3, 2005, pp. 46-49.

[6] S. L. Zhang, S. X. Fu and C. X. Li, "Structure and Function of Bigm Multi-Source Geology Information System [J],” Geology and Prospecting, Vol. 39, No. 3, 2003, pp. 63-66.

[7] X. C. Mao, L. Lin and C. Chen, "Design and Implementation of the General Geology Database Management System Based on Net Famework [J]," Contributions to Geology and Mineral Resources Research, Vol. 25, No. 2, 2010, pp. 164-166.

[8] X. Mao, T. G. Dai, Y. H. Zou and X. B. Wu, "Research and System Development of Geological Mineral Database of the Dachang Orefield [J]," Geology and Prospecting,
Vol. 39, No. 5, 2003, pp. 72-76.

[9] J. X. Huang, G. S. Bao and Z. D. Liu, "Interchange Software of Muti-Source Geoscientific Information System for Mineral Resources [J],” Journal of Central South University of Technology, Vol. 32, No. 3, 2001, pp. 227230.

[10] J. R. A. Giles, D. J. Lowe and K. A. Bain, "Geological Dictionaries-Critical Elements of Every Geological Database [M],” Computer \& Geoscience, Vol. 23, No. 6, 1997, pp. 621-626.

[11] N. Wang, H. B. Xu and N. B. Wang, "Data Tree-A Common Data Model for Integrating Heterogeneous Data Sources [J]," Computer Research \& Development, Vol. 35, No. 7, 1998, pp. 610-615. 